\title{
Effect of zinc supplementation on the growth of exclusively breastfed newborn babies
}

\author{
Bashir Ahmad Charoo, Aajaz Ahmad Mir, Ishtiyaq Ahmad Qadri, \\ Qazi Iqbal Ahmad, Javeed Iqbal Bhat
}

\begin{abstract}
:
Introduction: Zinc is an essential element, a component of several thousand enzymes as well as DNA \& RNA. Amongst the trace elements, the abundance of the zinc in the human body is second only to Iron hence playing an indispensable role in growth.

Aims and objectives: The aim was to study the prevalence of zinc deficiency in the newborn babies and the effect of zinc supplementation on their growth.

Materials and methods: This was an open label RCT conducted in SKIMS Srinagar, a tertiary care hospital in north India, from July 2013 to May 2015. The study group included all newborns who were exclusively breastfed. The babies were randomly divided into study group; who received $5 \mathrm{mg}$ OD of elemental zinc for first 6 months of life and control group who were exclusively breastfed. Zinc status was measured by serum zinc level and growth was ascertained by weight, length and head circumference at birth, 3 months and 6 months of age.

Results: A total of 60 neonates were studied, 32 cases and 28 controls. Baseline variables were comparable. Total of $45 \%$ of the babies were zinc deficient at birth. Zinc supplementation was found to further increase the length in all babies $(3.69 \pm 1.44 \mathrm{~cm})$ but more so in preterm babies $(4.25 \pm 1.73 \mathrm{~cm})$. Extra increment in the weight was only significant in preterm babies and head circumference showed no significant change.
\end{abstract}

\section{Introduction}

Zinc is an essential trace element second only to iron in abundance in the human body. Amongst its properties, the first to be identified were the catalytic properties. It is a component of thousands of enzymes as well as nucleic acids ${ }^{1}$. Adequate intake of zinc is critical for maintenance of epithelial and tissue integrity through promoting cell growth and suppressing apoptosis and through its underappreciated role as an antioxidant ${ }^{2}$. Thus, in the case of diarrhea, multiple functions of zinc may help to maintain the integrity of gut mucosa to reduce or prevent fluid loss ${ }^{3}$.

Global prevalence of zinc deficiency is estimated at 31\% ranging from 4-73\% across subregions ${ }^{4}$. Asymptomatic zinc deficiency, defined as serum zinc $<65 \mathrm{mcg} / \mathrm{kg}$, is most common followed by mild zinc deficiency. Growth retardation is perhaps the most common presentation of zinc deficiency. Organ systems affected clinically by severe zinc deficiency states include skin, gastrointestinal, CNS, immune, skeletal and reproductive systems. Acrodermatitis enteropathica, a rare autosomal disorder leading to inability to absorb zinc from intestine, is the most severe form of zinc deficiency ${ }^{5}$.

Good sources of zinc are meat, fish, eggs, milk, beans and nuts. Zinc is mainly absorbed from duodenum and appears to be dependent on a transport protein-metallothionein. The absorption of zinc is inhibited by dietary phytate, fiber, oxalate, iron and copper. Despite small RDA many people in both developing and industrialized countries do not have adequate zinc in their diet ${ }^{3}$.Zinc is used for treating acrodermatitis enteropathica, Zinc deficiency as well as in Wilson's disease. WHO \& UNICEF recommend that all should receive oral zinc for 10-14 days when they develop acute diarrhea ${ }^{6}$.

The EC directive guidelines recommend a zinc concentration of 300-1060 $\mu \mathrm{g} / 100 \mathrm{ml}$ of formula for term infants and the ESPGHAN committee recommended a daily intake of $1.1-2.0 \mathrm{mg} / \mathrm{kg}$ for preterm infants. The value of zinc supplementation for the long term growth and development of infants born preterm or small for gestational age requires further study ${ }^{2}$. A metanalysis indicated that daily zinc supplementation can reduce the incidence of pneumonia by $41 \%$ and diarrhea by $18 \%^{3}$. A trial of the daily zinc supplementation in otherwise healthy children from New Guinea reduced the number of malaria cases seen in the health clinics by $38 \%{ }^{7}$. Daily zinc supplementation in women with a relatively low plasma zinc concentration in in early pregnancy is associated with greater infant birth weight and head circumference ${ }^{8}$. Babies who are small for gestational age also seem to benefit from taking zinc supplementation ${ }^{8}$. A trial in India found that babies who received zinc from 1 month onwards were $60 \%$ less likely to die during infancy ${ }^{9}$. Lastly several studies indicate a potential role of zinc in improving immune status and health in elderly people ${ }^{9}$. 
Some evidence now indicates that as many as $25 \%$ cases of diarrhea and $40 \%$ cases of pneumonia can be prevented by preventing zinc deficiency ${ }^{10}$. LBW infants are at greater risk of zinc deficiency and its sequel. Improvement in brain function and physical growth velocity and diminution of the infectious disease morbidity has also been documented ${ }^{11}$. Zinc supplementation improves bone strength ${ }^{10}$. Zinc requirement increases during wound healing and zinc levels should be checked periodically in critically ill patients who fail to respond to nutritional support with adequate protein and calorie intake ${ }^{12}$.

Among the most urgent remaining issues are the identification of the population at most risk of zinc deficiency and identification of culturally appropriate and sustainable strategies to reduce the prevalence of zinc deficiency through the changes in the dietary practices ${ }^{13}$. This will in turn lead to improved growth as well as decreased morbidity and mortality ${ }^{14}$.

\section{Materials And Methods}

The study was conducted at SKIMS Srinagar, a tertiary care hospital in north India. This was an open label Randomized Controlled Trial conducted to know the effect on the growth of exclusively breastfed newborn babies by 6 months zinc supplementation. Subjects were randomly allocated to a study group; who were given 5mg OD of Zinc Sulphate and to a control group; who were exclusively breastfed over a period of 6 months. Both term and preterm babies who were in their first week of life, able to breastfeed and free from any disease or deformity were included in the study.

Baseline parameters were calculated at beginning of the study. Mother's zinc status was assessed by determining breastmilk Zinc concentration and baby's was determined by serum zinc concentration, at the beginning of study. Growth was monitored by measuring weight, length and head circumference; initially, at 3 months and at 6 months of age. The study was approved by IEC of SKIMS, Srinagar. Informed consent was taken from mother.

Serum zinc level was determined by colorimetric method and breastmilk zinc was determined by atomic absorption spectrometry.

Naked weight of the baby was determined by electronic weighting machine accurate up to last $10 \mathrm{~g}$. Length was measured by an infantometer where and head circumference was measured as the largest occipitofrontal diameter by a flexible but non-stretchable inch tape; both accurate to last $0.1 \mathrm{~cm}$.

\section{Results}

A total of 70 neonates were included in the study initially; among them 4 had introduced formula feeding, 3 had stopped giving zinc and 3 did not come for follow up. The study was finally conducted in a total of 60 neonates, 32 received Zinc and 28 served as controls. A sample size of 60 was found to be sufficient to detect a $20 \%$ difference in weight or length with alpha error of 0.05 and beta error of 0.20 . Statistical analysis was done by SPSS software (Version 20). The distribution of term and preterm babies in the two groups were equitable. We analyzed first categorical variables and continuous variables at birth to check for any significant difference which may confound the results of the study. Later we assessed the effect of our intervention on the growth by finding the increment at $3 \& 6$ months in weight, length and OFC.

Baseline variables including gestational age, age, mother's age, birth order, weight, length, headcircumference, serum zinc at birth and breast milk zinc were comparable in the two groups. Prevalence of zinc deficiency was found to be $45 \% .38$ were term and 22 were preterm. 38 were term and 22 preterm and 33 were male and 27 were female. Majority of them were of birth order 1 or 2 . Average age of babies was 3.5 days in the study group and 4.17 days in control group.

The extra gain in weight in the study group was significant only in the preterm babies after 6 months of the Zinc supplementation. There was a positive effect of zinc supplementation on the length, both at 3 months and at 6 months. Increment in the length $(4.25 \pm 1.73)$ in the preterm babies was more compared to the increment in whole study group. There was no significant greater gain in head circumference in the study group compared to the control group at any time in the study.

Table 1: Baseline variables at birth

\begin{tabular}{|l|l|l|l|}
\hline & ZINC GROUP(SD) & CONTROL GROUP & P VALUE \\
\hline AGE OF THE BABY & $3.50 \pm 1.73$ & $4.17 \pm 1.97$ & 0.20 \\
\hline BIRTH ORDER & $2.06 \pm 0.72$ & $1.46 \pm 0.63$ & 0.15 \\
\hline MOTHERS AGE & $30.50 \pm 3.17$ & $29.36 \pm 2.43$ & 0.12 \\
\hline GESTATIONAL AGE & $37.59 \pm 3.04$ & $36.71 \pm 3.03$ & 0.27 \\
\hline WEIGHT AT BIRTH & $2499 \pm 783$ & $2367 \pm 646$ & 0.48 \\
\hline LENGTH AT BIRTH & $47.33 \pm 4.34$ & $47.55 \pm 3.75$ & 0.83 \\
\hline HEAD CIRCUMFERENCE AT BIRTH & $33.00 \pm 2.43$ & $32.83 \pm 2.60$ & 0.79 \\
\hline SERUM ZINC AT BIRTH & $69.38 \pm 14.51$ & $67.43 \pm 15.98$ & 0.62 \\
\hline PREVALENCE OF ZINC DEFICIENCY & 47 & 43 & 0.71 \\
\hline BREAST MILK ZINC & $0.51 \pm 0.09$ & $0.50 \pm 0.12$ & 0.78 \\
\hline
\end{tabular}


Effect of zinc supplementation on the growth of exclusively breastfed newborn babies

Table 2: Effect of Zinc supplementation on the growth

\begin{tabular}{|c|c|c|c|c|c|c|c|}
\hline & & Weight (g) & $\mathrm{P}$ & Length $(\mathrm{cm})$ & $\mathrm{P}$ & $\mathrm{OFC}(\mathrm{cm})$ & $\mathrm{P}$ \\
\hline \multirow{2}{*}{ At birth } & Zinc group & $2499 \pm 783$ & \multirow[b]{2}{*}{0.48} & $47.33 \pm 4.34$ & \multirow[b]{2}{*}{0.83} & $33.00 \pm 2.42$ & \multirow[b]{2}{*}{0.79} \\
\hline & Control group & $2367 \pm 646$ & & $47.55 \pm 3.75$ & & $32.83 \pm 2.60$ & \\
\hline \multirow[t]{2}{*}{ At 3 months } & Zinc group & $4193 \pm 1008$ & \multirow[b]{2}{*}{0.21} & $57.03 \pm 4.42$ & \multirow[b]{2}{*}{0.04} & $39.95 \pm 1.85$ & \multirow[b]{2}{*}{0.12} \\
\hline & Control group & $3829 \pm 1214$ & & $54.93 \pm 4.81$ & & $38.22 \pm 2.35$ & \\
\hline \multirow[t]{2}{*}{ At 6 months } & Zinc group & $5958 \pm 1477$ & \multirow[b]{2}{*}{0.29} & $62.81 \pm 4.47$ & \multirow[b]{2}{*}{0.02} & $43.27 \pm 0.79$ & \multirow[b]{2}{*}{0.08} \\
\hline & Control group & $5571 \pm 1347$ & & $59.33 \pm 7.08$ & & $41.25 \pm 2.13$ & \\
\hline \multirow[t]{2}{*}{ Increase At 3 months } & Zinc group & $1694 \pm 496$ & \multirow[b]{2}{*}{0.22} & $9.70 \pm 2.88$ & \multirow[b]{2}{*}{0.01} & $6.95 \pm 3.07$ & \multirow[b]{2}{*}{0.11} \\
\hline & Control group & $1461 \pm 176$ & & $7.37 \pm 2.17$ & & $5.39 \pm 0.66$ & \\
\hline \multirow[t]{2}{*}{ Increase At 6 months } & Zinc group & $3458 \pm 885$ & \multirow[b]{2}{*}{0.31} & $15.47 \pm 5.21$ & \multirow[b]{2}{*}{0.013} & $10.26 \pm 5.10$ & \multirow[b]{2}{*}{0.61} \\
\hline & Control group & $3203 \pm 1077$ & & $11.77 \pm 5.96$ & & $8.41 \pm 1.68$ & \\
\hline Extra Increment At 3 months & Zinc group & $232 \pm 190$ & 0.22 & $2.32 \pm 0.65$ & 0.01 & $1.55 \pm 0.59$ & 0.11 \\
\hline \multirow[t]{2}{*}{ Extra Increment At 6 months } & Zinc group & $254 \pm 253$ & 0.31 & $3.69 \pm 1.44$ & 0.013 & $1.84 \pm 1.01$ & 0.61 \\
\hline & Preterm group & $317 \pm 124$ & 0.04 & $4.25 \pm 1.73$ & 0.01 & $2.11 \pm 0.95$ & 0.31 \\
\hline
\end{tabular}

\section{Discussion}

Many studies have indicated that a negative zinc balance exist in neonates especially in preterm neonates. Several workers have recorded an improved zinc status, good weight gain and better linear growth velocities in babies supplemented with zinc. Whether the effect of zinc is direct consequence of improved supply of this nutrient for growth and metabolism or is mediated through stimulation of appetite and/or less diarrheal episodes is unclear ${ }^{15,16}$. Zinc is essential for growth and even mild zinc deficiency leads to growth retardation. Global prevalence of zinc deficiency is around $31 \%(4-71 \%){ }^{17}$.

The current study was planned to evaluate the effects of zinc supplementation in growth. A number of studies were conducted both in developing and developed countries to find the effect of zinc supplementation on growth, but all of them were conducted in preterm or IUGR babies. Further they gave conflicting results with majority showing significant effects on growth and some showing no significant effect. Pedro IC Lira et al from Brazil in $1988^{18}$, Goldenberg et al from UK in $1995^{19}$, John A. Rivera et al from USA in $1998^{20,}$ Osendarp et al from Bangladesh in $2002^{21}$, Islam MN et al from Bangladesh in $2010^{22}$ and Aminsani et al from Iran in 2011 ${ }^{23}$ found significantly better growth after giving $5 \mathrm{mg}$, or more, of Zinc per day for a duration of 2 months. On the other hand Bueno $O$ et al from Italy in $2008^{24}$ did not find any significant effect on the growth after supplementing the IUGR babies with $3 \mathrm{mg} /$ day of zinc for first 6 months of life. The difference in the outcome was either because of differences in the quantity or because of the geographical and socioeconomic impact. We conducted a study in developing nation where zinc deficiency is rampant and gave adequate (5mg OD) of zinc for an adequate duration (first 6 months of life) to study the real impact of zinc supplementation in presence of high probability of zinc deficiency.

A high prevalence of zinc deficiency, $45 \%$, was found in our region; globally it varies from 4 to $71 \%{ }^{17}$. Growth was measured at the time of entry into study, at 3 and 6 months of age. At 3 months of age; no significant increment was found in head circumference but the increment in length was significant $(2.32$ $\mathrm{cm} \pm 0.65)$; further, weight gain was more in preterm babies $(317 \mathrm{~g} \pm 124)$. Roy et al in 1997 found that $2 \mathrm{months}$ of Zinc supplementation caused a significantly greater weight gain but the infants were also given dietary supplement ${ }^{25}$. Rivera et al in 1998 studied the effect of 3 months Zinc supplementation on the length of infants; He found to increase it by $1.4 \mathrm{~cm}^{20}$.

The study was concluded after 6 months of zinc supplementation. The effects on linear growth were most pronounced. Length increased by $15.47 \pm 5.21$ centimeters in the Zinc group and $11.77 \pm$ in the control group with an increment of $3.69 \pm 1.44$ centimeters in the study group, the p value was 0.013 . Among the babies who were given the zinc, increase in length $(4.25 \pm 1.73)$ was more in the preterm group than in those who were term, $\mathrm{p}=0.01$. Aminsani et al from Iran also studied the effect of 6 months zinc supplementation on the growth in preterm babies. They found significantly greater increase in length in the study group (16.9 $\pm 8.2 \mathrm{~cm}$ in the Zinc group compared the $15.1 \pm 4.1 \mathrm{~cm}$ in the placebo group, with absolute increment of $1.8 \pm 0.95 \mathrm{~cm}, \mathrm{p}=0.039$ ) 23. Our study showed more gain in the length, it may be because of the genetic and environmental factors. Ram Kumar TV found that zinc supplementation @ $10 \mathrm{mg}$ /day increased the length velocity in the study group by $0.31 \pm 0.12 \mathrm{~cm} /$ week more than in the control group in VLBW babies. The increase in length in 12 months was $5.2 \mathrm{~cm}$ length $(55.9 \pm 2.4 \mathrm{~cm}$ vs. $50.7 \pm 3.9 \mathrm{~cm}$, p value 0.001$)$, which is larger than our $4.25 \pm 1.73 \mathrm{~cm}$ may be because we supplemented zinc only for 6 months ${ }^{26}$.

In contrast, increment in weight gain was significant only in preterm babies $(317 \pm 124)$. There are no similar studies for term babies although Aminsani et al from Iran found significantly greater weight gain $(4995 \mathrm{~g} \pm 74 \mathrm{Vs} 3896 \mathrm{~g} \pm 865)$ after 6 months of zinc supplementation in preterm babies ${ }^{23}$.

Mild zinc deficiency does not seem to severely impair brain growth as zinc supplementation had no significant effect on the head circumference even in preterm babies. Other studies either showed minimal or insignificant gain in $\mathrm{HC}$. 
To conclude; zinc deficiency is very rampant in our part of the world; zinc deficiency can be prevented by zinc supplementation in at the risk preterm and IUGR babies. Zinc supplementation by $5 \mathrm{mg}$ OD of elemental zinc has been found to improve growth in preterm and IUGR babies however bigger studies need to be done before making zinc supplementation a routine for its effect on the growth, acute gastroenteritis and respiratory tract infections.

\section{Bibliography}

[1]. www.wikipedia.org/wiki/zinc

Rennie and Robertson's, Textbook of Neonatology $5^{\text {th }}$ edition: 826-827.

[2]. Rennie and Robertson's, Textbook of Neona

[4]. Brown et al, food and nutrition Bulletin 2001: 22; 113-125.

[5]. Hambridge and Mcleran, Am J Clin Nutr 1982; 36:943-48.

[6]. Nelsons Textbook of Pediatrics, $19^{\text {th }}$ edition, 2328-2329.

[7]. $\quad$ Shaker AH et al, Am J Trop Med \& Hyg: 663-669.

[8]. Goldenberg RL, JAMA 1995; 463-468.

[9]. Fortes C et al, Am Geriatric Soc 1998; 46(1): 19-26.

[10]. www.nlm.nih.gov/..982.html.

[11]. Polin, Fox and Abman, Fetal and Neonatal Physiology $4^{\text {th }}$ edition: 403-408

[12]. Rogers, Pediatric Critical Care $4^{\text {th }}$ edition 1511.

[13]. Walker and goulet, Pediatric Gastrointestinal Diseases, 5th edition: 321-327

[14]. Evan Mayo-Wilson et al, Cochrane Library, 2014

[15]. Itabashi K \& Saito; Boil Neonate 2003; 83: 235-240.

[16]. Hanbidge KM et al, Zinc in the fetus and Neonate; Paulin, Fox and Abman; Fetal and Neonatal Physiology.

[17]. Laura and Black, Comparative Quantification of health risks, pp 27-279.

[18]. Am J Clin Nutr 1988; 68(Suppl): 4185-245.

[19]. JAMA; 1995 Aug 9; 274(6): 463-8.

[20]. J Nutr 128: 556-562, 1998.

[21]. Am J Clin Nutr 2002; 76: 1401-1408.

[22]. Indian Pediatr 2010 Oct; 47(10): 845-849.

[23]. Indian J Pediatr. 2011 Oct; 78(10): 1239-43.

[24]. Nutr Hosp.2008 May-Jun; 23 (3): 212-219.

[25]. Roy et al, Arch Dis Child, 1997 Sept 77(3); 196-200.

[26]. J Pediatrics. 2003 May; 111(5p+1)1002-9. 